\title{
Platelet RNA signatures for the detection of cancer
}

\author{
Nik Sol ${ }^{1,2}$ Thomas Wurdinger ${ }^{2,3,4}$
}

Published online: 5 July 2017

(C) The Author(s) 2017. This article is an open access publication

\begin{abstract}
Platelets are equipped with RNA processing machineries, such as pre-mRNA splicing, pre-miRNA processing, and mRNA translation. Since platelets are devoid of a nucleus, most RNA transcripts in platelets are derived from megakaryocytes during thrombocytogenesis. However, platelets can also ingest RNA molecules during circulation and/or interaction with other cell types. Since platelets were first described by Bizzozero in 1881, their well-established role in hemostasis and thrombosis has been intensively studied. However, in the past decades, the list of biological processes in which platelets play an important role keeps expanding. In this review, we discuss how platelet RNA biomarker signatures can be altered in the presence of cancer.
\end{abstract}

Keywords Platelets $\cdot$ Transcriptome $\cdot$ mRNA $\cdot$ Splicing · Tumor-educated platelets $\cdot$ Liquid biopsy $\cdot$ Biomarkers

Nik Sol

ni.sol@vumc.nl

Thomas Wurdinger

t.wurdinger@vumc.nl

1 Department of Neurology, VU University Medical Center, Amsterdam, The Netherlands

2 Brain Tumor Center Amsterdam, VU University Medical Center, Amsterdam, The Netherlands

3 Department of Neurosurgery, VU University Medical Center, Amsterdam, The Netherlands

4 Department of Neurology, Massachusetts General Hospital and Neuroscience Program, Harvard Medical School, Boston, MA, USA

\section{Platelets and cancer in brief}

Platelets are implicated in tumor biology and metastasis (Fig. 1) [1,2], and tumor cells can, directly and indirectly, impose changes on platelet RNA and protein content [3-5]. As a result, these tumor-educated platelets (TEPs) have an altered function and can in various ways promote tumor cell survival and metastasis, as well as other hallmarks of cancer [6]. Tumor cells interact with platelets indirectly via different signaling molecules or directly via different receptors, mainly the platelet activation receptor P-selectin [7-10]. Upon activation, platelets can release several growth and pro-angiogenic factors like ANGPT1, PDGF, BFGF, EGF, HGF, IGF1, TGF $\beta$, VEGF-A, and VEGF-C $[11,12]$. Platelets can release these factors at a metastatic niche, thereby providing a pro-tumoral growth microenvironment [1].

Calverley et al. showed that NAD-dependent deacetylase sirtuin-2 (SIRT2) is differentially spliced in platelets of metastatic lung cancer patients. This gene plays a role in epigenetic silencing, concluding that platelets could induce growth and progression of tumors by releasing epigenetic silencers [5]. Further, platelets alter cancer cells to evade the detection by the immune system, by transferring MHC class I proteins to the tumor cells, resulting in protection against natural killer cells [13]. Platelets can also mechanically protect cancer cells from destruction via NK cells by forming a cell-fibrin-platelet aggregate surrounding circulating tumor cells (CTC) or arrested tumor cells. This physical shield avoids cellmediated immune detection and supports cancer cell survival [14-17]. Such protective properties of platelets are important for cancer metastasis by promoting cancer cell survival in the circulation $[18,19]$. 

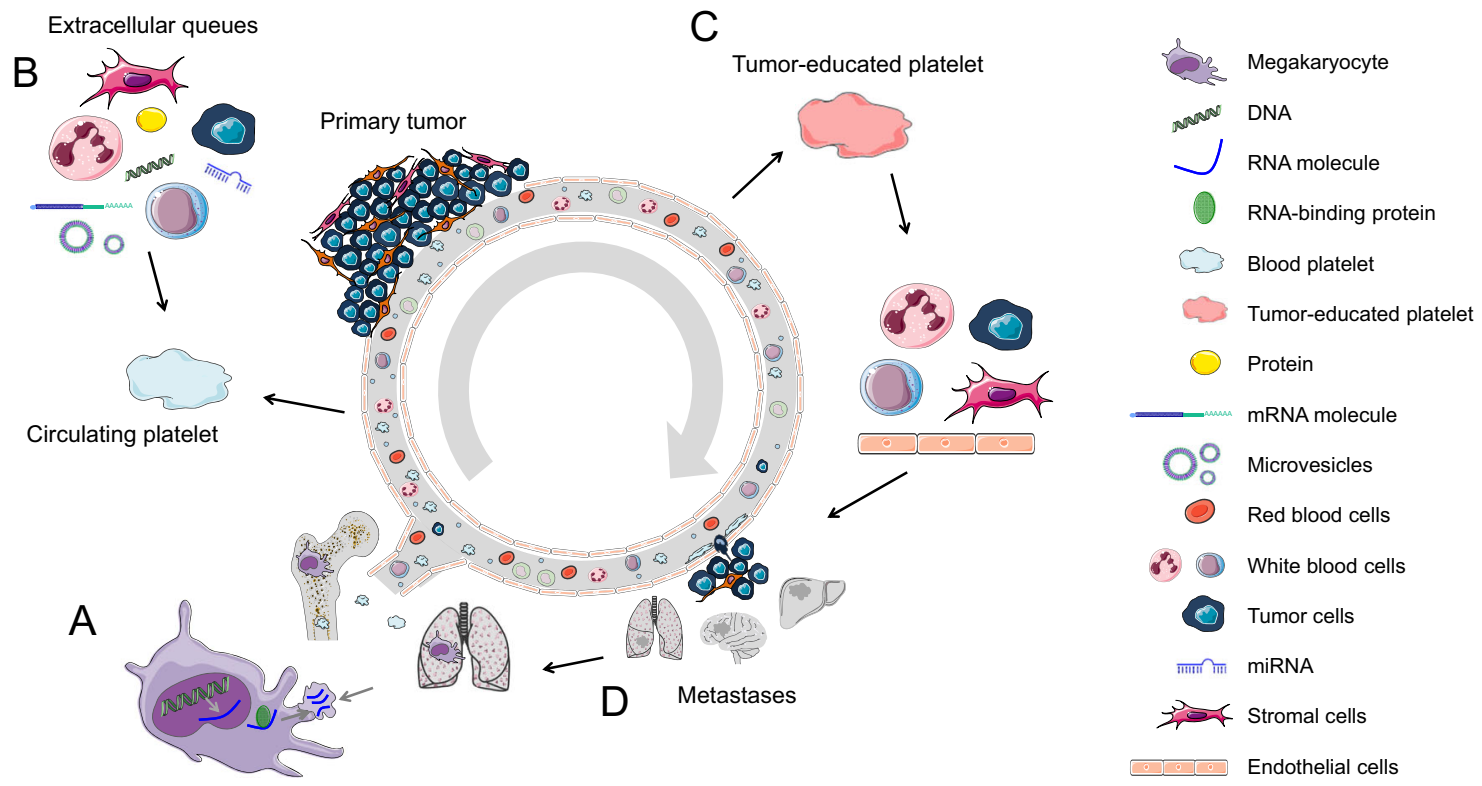

Fig. 1 Schematic representation of tumor-mediated education of platelets and the megakaryocyte leading to metastasis. (a) Megakaryocytes in the bone marrow and lungs sort specific RNA and proteins into platelet precursors. (b) Circulating platelets contain a variety of RNA transcripts and proteins. During their 7-10-day lifespan, platelets interact with immune cells, cancer cells, and stromal cells. These direct interactions as well as distant cell signaling, for instance, via vesicle-mediated

\section{Liquid biopsies and cancer detection}

Liquid biopsies have been introduced as a potential game changer in cancer management, with blood tests emerging as a minimally invasive, safe, and sensitive alternative or complementary approach for tissue biopsies [20-22]. Blood represents a rich source of information through which solid cancers (and their subtypes) can be detected, identified and classified, and matched to a specific therapy [23-30]. Targeted risk-based screening based on a person's individual risk of cancer is envisioned to be the anti-cancer strategy of the future. Current clinical oncology practice relies on the removal of tumor tissue through biopsies for analysis of tumor-linked genetic alterations. Although the use of tumor tissue biopsies is the current gold standard for cancer diagnosis and represents an essential tool in cancer management, it has become apparent that the information acquired from a single biopsy provides a spatially and temporally limited snapshot of a (metastatic) tumor and often fails to reflect the heterogeneity of the disease [22, 31, 32]. Moreover, tumor biopsies are invasive which poses a limitation for repeated sampling (needed for monitoring treatment response and resistance to targeted therapies). Liquid biopsies could provide a potential revolution in cancer diagnostics as a minimally invasive method for detecting and monitoring diseases, complementary to current tissue biopsy approaches. Liquid biopsies can therefore provide an accurate and comprehensive spatiotemporal snapshot communication in whole blood, changes the content of the platelet and platelet function. (c) This process leads to the development of tumoreducated platelets. Next, tumor-educated platelets can influence the process of metastasis formation by stimulating or blocking immune cells, endothelial cells, stromal cells, and cancer cells, either by direct cell-to-cell contact or by releasing extracellular queues. (d) Finally, metastasis could affect the sorting of specific RNA and proteins of megakaryocytes into platelets

of the tumor and its microenvironment on multiple levels, and enable (1) early detection (screening), (2) prognosis for the individual patient: stage and spread, (3) identification of new targets for personalized treatment, (4) pre-treatment classification for personalized therapy/prediction of response to therapy, (5) early therapy response monitoring, "real-time" assessment of treatment effectiveness, and (6) follow-up and early detection of recurrence of the disease and its metastases. Currently, blood-based biopsy measurements focus on evaluation of biomarker biosources, including circulating tumor DNA (ctDNA), circulating tumor cells (CTCs), extracellular vesicles (EVs; exosomes, microvesicles, microparticles, oncosomes), and tumor-educated platelets (TEPs) [3, 4, 33-38].

\section{Tumor-educated platelets}

Platelets have long been considered as a potential diagnostic tool in cancer. Several studies have shown that a simple platelet count already harbors potential clinical relevant information [39-44]. Besides platelet counts, the size of platelets [44, 45] and platelet protein markers, such as P-selectin, are used for blood-based cancer diagnostics and prognostics [44, 46-51]. Platelets can interact with cancer cells in various ways leading to platelet hyper-reactivity $[52,53]$. Furthermore, there may be an increase in young reticulated platelets in 
cancer patients [54]. The ratio of these young platelets in the total platelet population can change again after cancer treatment $[43,48,55]$. These observations indicate that platelets can respond reactively during tumor progression and treatment.

Tumor-associated biomolecules are transferred to platelets resulting in their "education" $[3,4,56]$. External stimuli, such as activation of platelet surface receptors and lipopolysaccharide-mediated platelet activation, induce specific splicing of pre-messenger RNAs (mRNAs) in circulating TEPs [57]. TEPs may also undergo queue-specific splice events in response to signals released by cancer cells and the tumor microenvironment such as by stromal and immune cells [1]. The combination of specific splice events in response to external signals and the capacity of platelets to directly ingest (spliced) circulating mRNA can provide TEPs with a highly dynamic mRNA repertoire, with potential applicability to cancer diagnostics. A highly sensitive method for isolation and analysis of TEP RNA was developed to detect cancer. It was shown that platelet mRNA profiles can be used to distinguish between healthy donors and cancer patients [3-5]. Given the many roles platelets play in cancer, they could harvest information about the disease status of given patients.

Platelets are capable of taking up protein and nucleotides during their lifespan. Nilsson et al. described the uptake of extracellular vesicles (EVs) by circulating platelets [3]. Via this mechanism, platelets can sequester EVs from cancer cells harboring tumor-specific RNA. EGFRvIII, a deletion mutant of the epidermal growth factor receptor (EGFR), is such a specific tumor RNA which is considered to be present in $30 \%$ of glioblastoma tumors. Traces of this very malignant tumor of the central nervous system could be detected by RT-PCR of platelets from these patients. The EGFRvIII RNA transcript was detected with a sensitivity of $80 \%$ (four out of five EGFRvIII-positive tumors were detected), and a specificity of $96 \%$ (25 out of 26 EGFRvIII-negative tumors were scored as negative). In addition, microarray analysis discovered an RNA signature that could distinguish between glioblastoma patients $(n=8)$ and healthy controls $(n=12)$ [3]. A total of 17 out of the top 30 differentially expressed genes were also found in the mRNA sequencing data from TEPs of GBM patients, with four of the most significantly differentially expressed, WFDC1, FKBP5, IL1R2, and TPCN1. In 2015, the uptake of tumor-derived RNA in platelets was confirmed in NSCLC patients. Translocated EML4-ALK transcripts and KRAS and EGFR transcripts harboring tumorspecific point mutations were detected by RT-PCR and deep amplicon sequencing $[4,33]$. Although shallow sequencing of platelets did not reveal the specific mutations, the RNA profiles allowed for the development of surrogate gene panels to determine the molecular status of the tumor tissue in situ.

\section{TEP RNA signatures}

Microarray mRNA platelet profiles of seven healthy donors and five treatment-naive metastatic lung cancer patients were compared by Calverley et al. [5]. Unsupervised hierarchical clustering revealed 200 altered RNAs, of which all but 3 were decreased in platelets of lung cancer patients. They also showed that NAD-dependent deacetylase sirtuin-2 (SIRT2) is differentially spliced in platelets of metastatic lung cancer patients compared to healthy individuals. SIRT2 is a human homolog to yeast Sir2 protein that regulates epigenetic gene silencing and suppresses rDNA recombination and is a cellular stress response. In yeast, it regulates the acetylation status of several tumor suppressors including p53 and FoxO1; however, in humans its exact function is not yet known.

A recent study demonstrated that mRNA sequencing of tumor-educated platelets distinguishes cancer patients from healthy individuals with $96 \%$ accuracy (Fig. 2) [4]. Blood contains 200-500 million platelets per milliliter, making platelets highly available for diagnostic use. Whole blood can be stored up to $48 \mathrm{~h}$ at room temperature prior to platelet isolation while maintaining high-quality RNA and the dominant cancer RNA signatures. Platelet isolation was performed with a twostep centrifugation protocol, and platelet purity was confirmed by manual cell counting using crystal violet staining on a light microscope showing one to five nucleated cells per 10 million platelets. Nucleated cells are considered to contain approximately $10,000 \times$ more RNA than a single platelet. However, nucleated cell counts of one to five nucleated cells per 10 million platelets indicate that the RNA profiles that were observed in the platelet fraction are likely not attributable to the presence of nucleated cell contamination. Furthermore, in silico analysis showed a high correlation between TEP RNA profiles with previously described platelet RNA profiles, which were derived from platelet isolations using a CD45 depletion step to further diminish possible leukocyte contamination, and no correlation with nucleated blood cells (Fig. 3). In addition, based on DAVID Gene Ontology analysis, the detected RNAs are strongly enriched for transcripts associated with blood platelets. Among the 5003 RNAs, known platelet markers were identified, such as B2M, PPBP, TMSB4X, and PF4 in high levels [4].

RNA sequencing was performed on 283 blood platelet samples, isolated from healthy individuals $(n=55)$ and patients with cancer ( $n=228$, cancer types: glioblastoma, nonsmall cell lung cancer, colorectal cancer, pancreatic cancer, breast cancer, and liver and bile duct carcinoma) [4]. A total of 1453 out of 5003 mRNAs were increased and 793 out of 5003 mRNAs were decreased in TEPs as compared to platelet samples of healthy donors while presenting a strong correlation between these platelet mRNA profiles. Unsupervised hierarchical clustering based on the differentially detected platelet mRNAs distinguished two sample groups with minor 


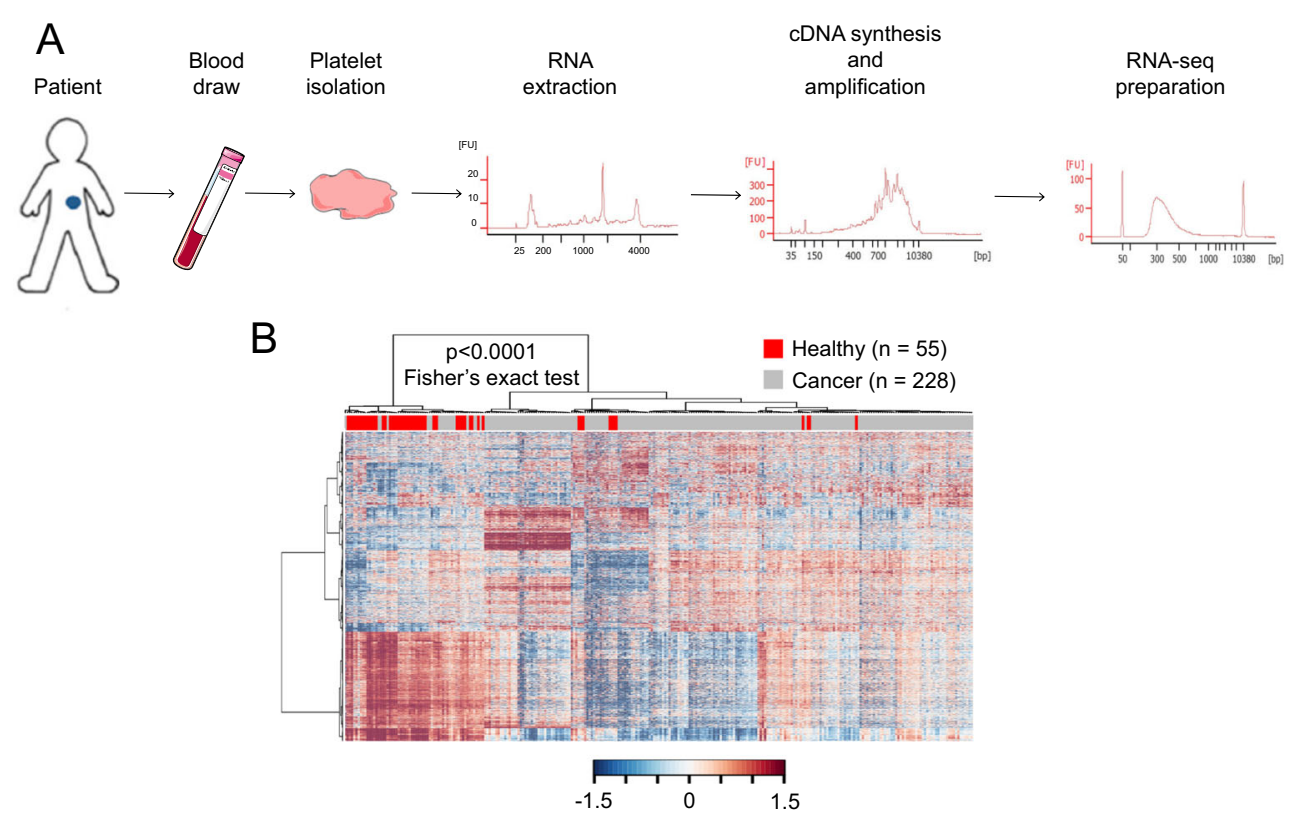

Fig. 2 ThromboSeq workflow. (a) TEP mRNA sequencing workflow; blood draw is performed on patients, and from a single 6-ml EDTAcoated tube, platelets are isolated. RNA extraction is done according to manufacturer's protocol using the mirVana RNA isolation kit (Life Technologies). Next, mRNA is amplified using SMARTer Ultra low input RNA kit (Clontech). Samples are prepared for sequencing on the
Hiseq 2500 Illumina platform using the Truseq Nano DNA Sample Prep Kit (Illumina). After each step, quality control was performed by critical inspection of Bioanalyzer profiles (Agilent). (b) Heatmap of unsupervised clustering of platelet mRNA profiles of healthy donors (red, $n=55$ ) and patients with cancer (gray, $n=228$ )

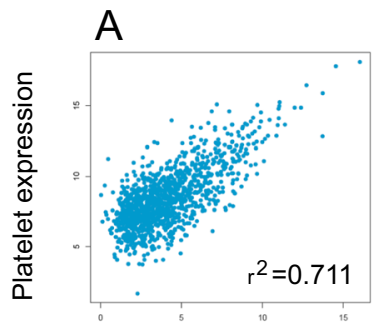

$\mathrm{F}$
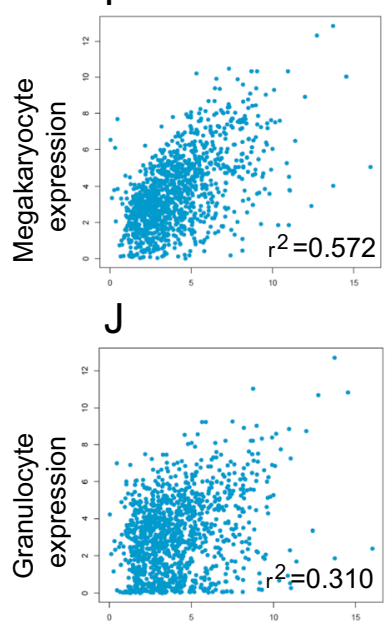

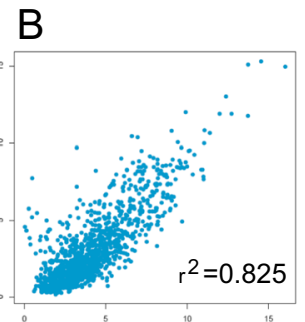

G
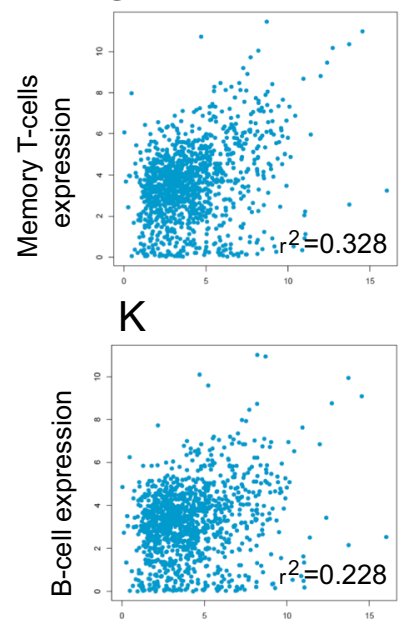
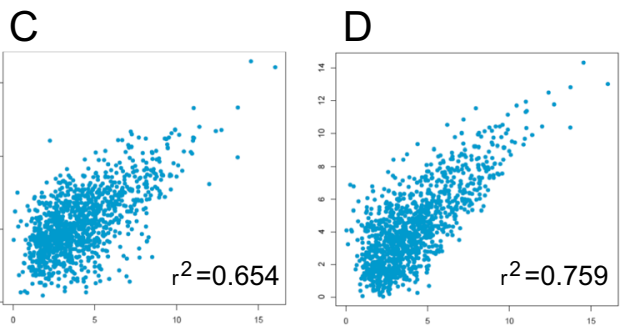

E
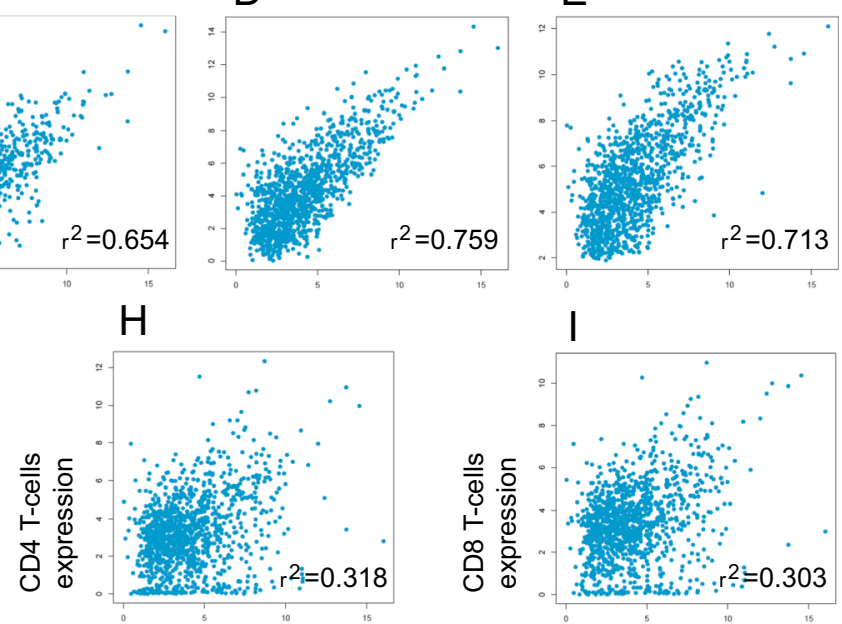

$\mathrm{L}$

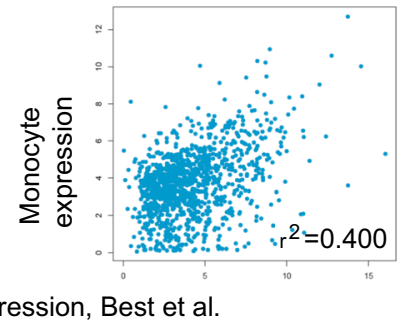

$\mathrm{M}$

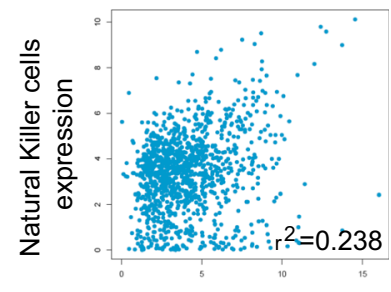

TEP + HC expression, Best et al
Fig. 3 Correlation plots of TEP RNA signatures with other (a) nucleated blood cells. Correlation plots between platelets sequenced by Best et al. compared with platelet RNA expression levels from different studies and compared with RNA expression from different blood cells. (a) Platelets from Bray et al. [82]. (b) Poly A selected RNAs from platelets from
Kissopoulou et al. [83]. (c) Ribosomal RNA-depleted platelets from Kissopoulou et al. [83]. (d) Platelets from Rowley et al. [84]. (e) Platelets from Simon et al. [85]. (f) Megakaryocyte [86]. (g) Memory T-cells [87]. (h) CD4 T-cells [87]. (i) CD8 T-cells [87]. (j) Granulocytes [87]. (k) B-cells [87]. (l) Monocytes [87]. (m) Natural killer cells [87] 
overlap. Using the different mRNA profiles of cancer patients and healthy donors, it was possible to develop a predictive algorithm with high accuracy in separating healthy individuals from cancer patients. The RNA profiles allowed for support vector machine (SVM) classification, enabling the ability to correctly classify whether the patient has cancer or not (accuracy: $96 \%$ ), which tumor type is present (accuracy $71 \%$ ), and which molecular mutational subtype the tumor has (accuracy 85-95\%). Interestingly, all 39 patients with early-stage, nonmetastasized cancer were correctly identified as cancer patients [4]. These promising data show that the TEP cancer classification platform deserves thorough follow-up evaluation experiments, profiling of additional prospectively collected patient cohorts, cancer (molecular) subtypes, and other (inflammatory) diseases, and further training and development of the cumulative SVM algorithms. This patient cohort included six (heterogenic) tumor types, i.e., non-small cell lung carcinoma $(n=60)$, colorectal cancer $(n=41)$, glioblastoma $(n=39)$, pancreatic cancer $(n=35)$, hepatobiliary cancer $(n=14)$, and breast cancer $(n=39)$.

Although it was not possible to measure significant differences between localized and metastasized tumors, it is not excluded that a larger sample set of localized and metastasized samples from the same cancer type will have the power to do so. Since the TEP profiles closely resemble the different tumor types as determined by their organ of origin, regardless of systemic dissemination, it was possible to develop a multiclass algorithm predicting the primary tumor location and separate tumors based upon their mutational status [4]. Different primary tumors showed different RNA profiles, making it possible to determine malignant lesions as a primary tumor or as a metastasis, for instance on chest imaging. Resemblance in platelet RNA profiles from different primary tumors is partly explained by similar driver mutations. KRAS for instance is a driver mutation in many cancer types. This mutation and others leave a specific signature in platelets making it possible to select patients for different targeted therapies. Furthermore, when sequenced deep enough, the specific driver mutations can be found in platelets.

This thromboSeq technique shows the potential of platelets as liquid biopsy biosource. Besides mRNA, platelets also contain non-coding and small RNAs. Analysis of differentially expressed non-coding RNAs revealed 20 genes of which 16 were upregulated in TEPs (Table 1). These 20 non-coding RNAs showed a tumor-specific profile. Interestingly, one of the downregulated RNAs is Metastasis Associated Lung Adenocarcinoma Transcript 1 (MALAT1) [4]. This noncoding RNA is retained in the nucleus where it acts as a transcriptional regulator of numerous genes, including some genes involved in cancer metastasis. Its upregulation in multiple cancerous tissues has been associated with the proliferation and metastasis of tumor cells [58-60]. Growth arrestspecific transcript 5 (GAS5), another downregulated non- coding RNA, is involved in cellular proliferation, and its downregulation has been shown to be pro-cancerous in several tumor types [61, 62]. Both SNHG5 (small nucleolar RNA host gene) and SNHG8 appear to play a role in gastric cancer by regulating migration and proliferation [63-65]. Lymphocytic leukemia 1 and 2 (DLEU1 and DLEU2) are frequently deleted in several hematological cancers, and cancer susceptibility candidate 15 (CASC15) has a role in the formation of neuroblastoma [66-68]. Given the function that these non-coding RNAs seem to have in cancer, it is interesting to further investigate non-coding RNA expression in platelets from cancer patients.

Of the non-coding RNAs, microRNAs are of special interest. Ple et al. described in 2012, 532 different micro-RNAs in platelets [69]. Increasing work suggests an important role for platelet microRNAs in platelet biogenesis and function [70-73]. In cardiovascular disease, nine differentially expressed microRNAs were found comparing patients with myocardial infarction with healthy controls [74]. This shows the potential of microRNAs in platelets as a diagnostic tool. Although microRNAs play an important role in cancer, their expression is mostly studied in tissue and exosomes. MicroRNA expression in TEPs needs further research to determine their diagnostic power.

DAVID and CAGE gene ontology algorithms were applied to the diagnostic RNA panels of tumor-educated platelets. This revealed a downregulation of RNAs involved in RNA metabolism and RNA splicing. Interestingly, there was a correlation to platelet activation, platelet and vesicle transport, cytoskeleton activation, and ATP signaling. These programs potentially reveal a hyperactive state of tumor-educated platelets matching the hyper-reactivity found in a functional analysis of platelets from certain cancer patients. This hyperactivity seems to be different in cancer patients compared to patients with non-cancerous inflammatory diseases. Microarray studies performed on platelets from different non-cancerous inflammatory diseases identified 22 differentially expressed genes [75-78]. Expression levels of these genes in TEPs appeared to be randomly expressed compared to platelets of healthy donors, suggesting that platelet RNA in patients with non-cancerous disease is different from patients with cancer [4]. More studies comparing TEPs with platelets from patients with non-cancerous inflammatory disease and healthy individuals could give us more insight into the function of platelets in different diseases including cancer. To gain more insight, the collection has been initiated of relevant patient samples from multiple non-cancerous clinical conditions, such as multiple sclerosis, inflammatory bowel disease, chronic obstructive pulmonary disease, cardiovascular disease, pancreatitis, and premalignant lesions (e.g., pancreatic intra-epithelial neoplasms). These cohorts may improve the strength of thromboSeq in the clinical practice by not only comparing healthy from cancer but separating patients with similar 


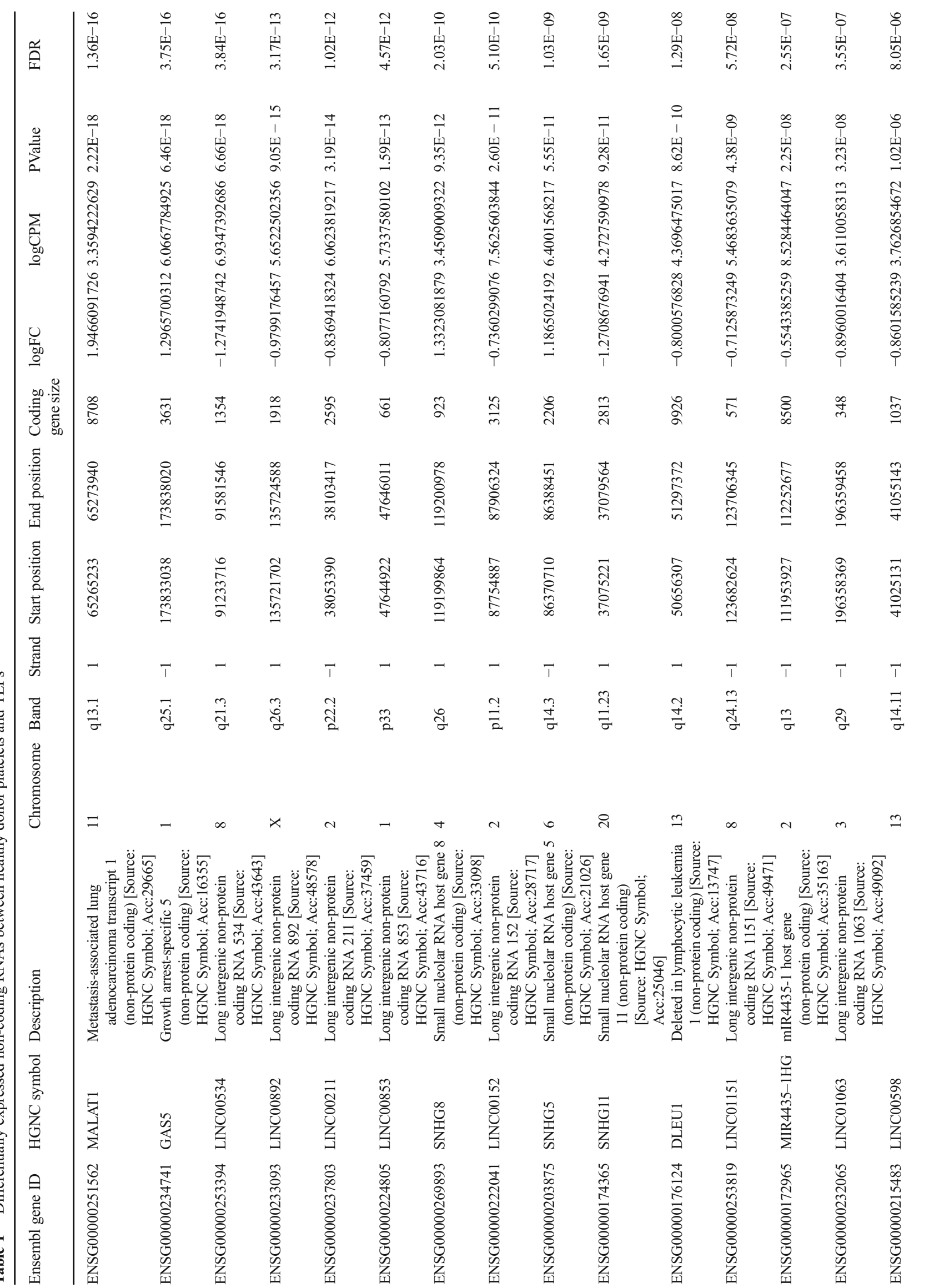




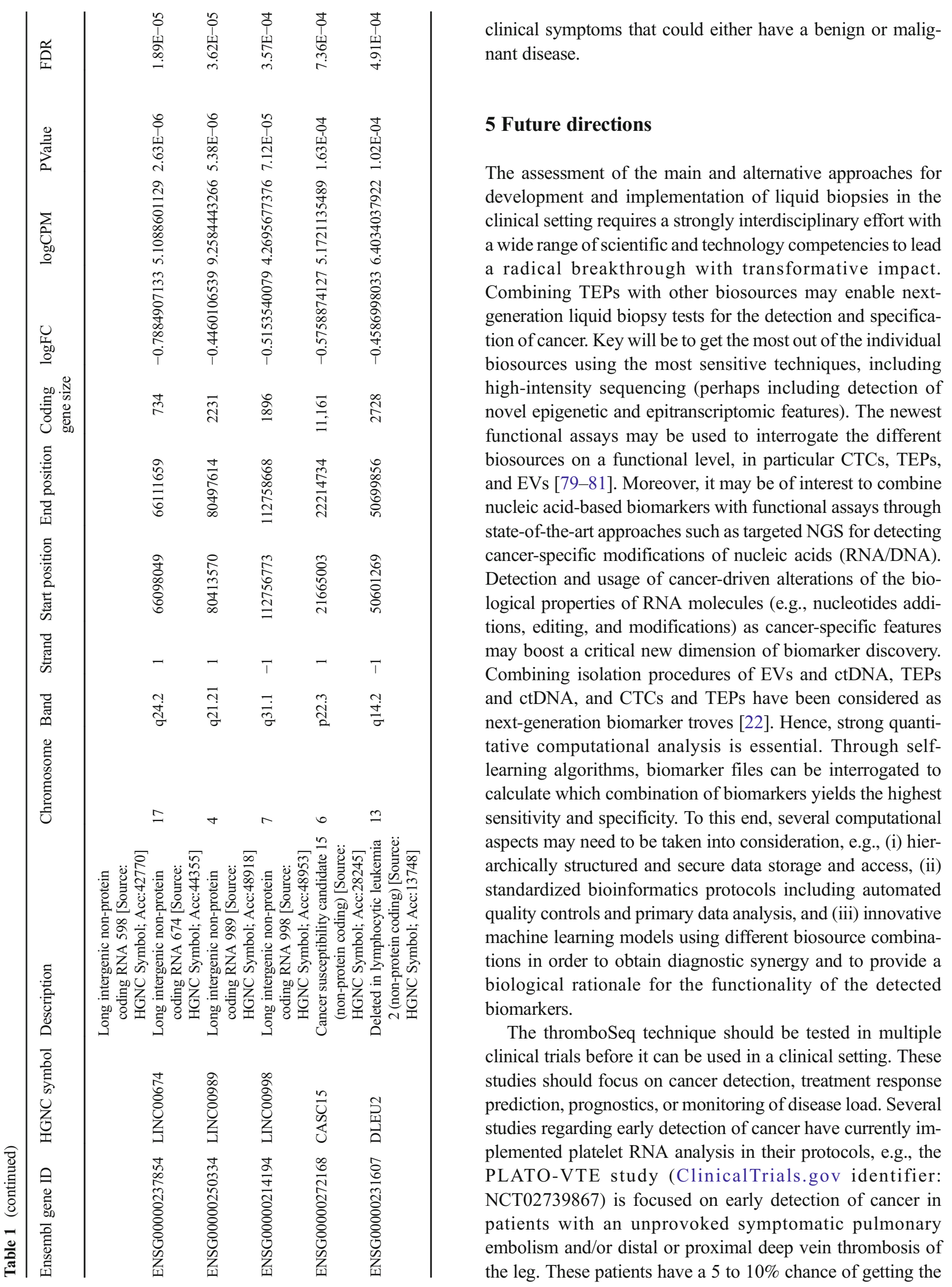


diagnosis of cancer within 1 year after the event. In the VTEPLATO study, platelet RNA will be analyzed to determine if cancer can be detected earlier, when cure rates are higher.

Open Access This article is distributed under the terms of the Creative Commons Attribution 4.0 International License (http:// creativecommons.org/licenses/by/4.0/), which permits unrestricted use, distribution, and reproduction in any medium, provided you give appropriate credit to the original author(s) and the source, provide a link to the Creative Commons license, and indicate if changes were made.

\section{References}

1. McAllister, S. S., \& Weinberg, R. A. (2014). The tumour-induced systemic environment as a critical regulator of cancer progression and metastasis. Nature Cell Biology, 16, 717-727.

2. Kuznetsov, H. S., et al. (2012). Identification of luminal breast cancers that establish a tumor-supportive macroenvironment defined by proangiogenic platelets and bone marrow-derived cells. Cancer Discovery, 2, 1150-1165.

3. Nilsson, R. J. A., et al. (2011). Blood platelets contain tumorderived RNA biomarkers. Blood, 118, 3680-3683.

4. Best, M. G., et al. (2015). RNA-Seq of tumor-educated platelets enables blood-based pan-cancer, multiclass, and molecular pathway cancer diagnostics. Cancer Cell, 28, 666-676.

5. Calverley, D. C., et al. (2010). Significant downregulation of platelet gene expression in metastatic lung cancer. Clinical and Translational Science, 3, 227-232.

6. Hanahan, D., \& Weinberg, R. A. (2011). Hallmarks of cancer: the next generation. Cell, 144, 646-674.

7. Borsig, L. (2008). The role of platelet activation in tumor metastasis. Expert Review of Anticancer Therapy, 8, 1247-1255.

8. Steinert, B. W., Tang, D. G., Grossi, I. M., Umbarger, L. A., \& Honn, K. V. (1993). Studies on the role of platelet eicosanoid metabolism and integrin alpha IIb beta 3 in tumor-cell-induced platelet aggregation. International Journal of Cancer, 54, 92-101.

9. Zucchella, M., et al. (1989). Human tumor cells cultured "in vitro" activate platelet function by producing ADP or thrombin. Haematologica, 74, 541-545.

10. Egan, K., et al. (2011). Platelet adhesion and degranulation induce pro-survival and pro-angiogenic signalling in ovarian cancer cells. PloS One, 6, e26125.

11. Menter, D. G., et al. (2014). Platelets and cancer: a casual or causal relationship: revisited. Cancer Metastasis Reviews, 33, 231-269.

12. Sierko, E., \& Wojtukiewicz, M. Z. (2004). Platelets and angiogenesis in malignancy. Seminars in Thrombosis and Hemostasis, 30, 95-108.

13. Placke, T., et al. (2012). Platelet-derived MHC class I confers a pseudonormal phenotype to cancer cells that subverts the antitumor reactivity of natural killer immune cells. Cancer Research, 72, 440-448.

14. Borsig, L., et al. (2001). Heparin and cancer revisited: mechanistic connections involving platelets, $\mathrm{P}$-selectin, carcinoma mucins, and tumor metastasis. Proceedings of the National Academy of Sciences of the United States of America, 98, 3352-3357.

15. Palumbo, J. S., et al. (2005). Platelets and fibrin(ogen) increase metastatic potential by impeding natural killer cell-mediated elimination of tumor cells. Blood, 105, 178-185.

16. Palumbo, J. S., et al. (2007). Tumor cell-associated tissue factor and circulating hemostatic factors cooperate to increase metastatic potential through natural killer cell-dependent and-independent mechanisms. Blood, 110, 133-141.

17. Labelle, M., Begum, S., \& Hynes, R. O. (2011). Direct signaling between platelets and cancer cells induces an epithelialmesenchymal-like transition and promotes metastasis. Cancer Cell, 20, 576-590.

18. Buergy, D., Wenz, F., Groden, C., \& Brockmann, M. A. (2012). Tumor-platelet interaction in solid tumors. International Journal of Cancer, 130, 2747-2760.

19. Gasic, G. J., Gasic, T. B., \& Stewart, C. C. (1968). Antimetastatic effects associated with platelet reduction. Proceedings of the National Academy of Sciences of the United States of America, 61, 46-52.

20. Alix-Panabières, C., \& Pantel, K. (2016). Clinical applications of circulating tumor cells and circulating tumor DNA as liquid biopsy. Cancer Discovery, 6, 479-491.

21. Diaz, L. A., \& Bardelli, A. (2014). Liquid biopsies: genotyping circulating tumor DNA. Journal of Clinical Oncology, 32, 579-586.

22. Bardelli, A., \& Pantel, K. (2017). Liquid biopsies, what we do not know (yet). Cancer Cell, 31, 172-179.

23. Chu, D., et al. (2016). ESR1 mutations in circulating plasma tumor DNA from metastatic breast cancer patients. Clinical Cancer Research, 22(4), 993-999.

24. Guttery, D. S., et al. (2015). Noninvasive detection of activating estrogen receptor 1 (ESR1) mutations in estrogen receptor-positive metastatic breast cancer. Clinical Chemistry, 61(7), 974-82.

25. Schiavon, G., et al. (2015). Analysis of ESR1 mutation in circulating tumor DNA demonstrates evolution during therapy for metastatic breast cancer. Science Translational Medicine, 7(313), 313 ra182.

26. Gorges, T. M., et al. (2016). Heterogeneous PSMA expression on circulating tumor cells: a potential basis for stratification and monitoring of PSMA-directed therapies in prostate cancer. Oncotarget, 7, 34930-34941.

27. Gorges, T. M., et al. (2016). Enumeration and molecular characterization of tumor cells in lung cancer patients using a novel in vivo device for capturing circulating tumor cells. Clinical Cancer Research, 22(9), 2197-206.

28. Maheswaran, S., et al. (2008). Detection of mutations in EGFR in circulating lung-cancer cells. The New England Journal of Medicine, 359, 366-377.

29. Miyamoto, D. T., et al. (2012). Androgen receptor signaling in circulating tumor cells as a marker of hormonally responsive prostate cancer. Cancer Discovery, 2(11), 995-1003.

30. Antonarakis, E. S., et al. (2014). AR-V7 and resistance to enzalutamide and abiraterone in prostate cancer. The New England Journal of Medicine, 371, 1028-1038.

31. Siravegna, G., et al. (2015). Clonal evolution and resistance to EGFR blockade in the blood of colorectal cancer patients. Nature Medicine, 21, 795-801.

32. Gerlinger, M., Norton, L., \& Swanton, C. (2013). Acquired resistance to crizotinib from a mutation in CD74-ROS1. The New England Journal of Medicine, 369, 1172-1173.

33. Nilsson, R. J. A., et al. (2015). Rearranged EML4-ALK fusion transcripts sequester in circulating blood platelets and enable blood-based crizotinib response monitoring in non-small-cell lung cancer. Oncotarget, 7, 1066-1075.

34. Schwarzenbach, H., \& Pantel, K. (2015). Circulating DNA as biomarker in breast cancer. Breast Cancer Research, 17, 136.

35. Alix-Panabières, C., \& Pantel, K. (2013). Circulating tumor cells: liquid biopsy of cancer. Clinical Chemistry, 59, 110-118.

36. Alix-Panabières, C., \& Pantel, K. (2014). Challenges in circulating tumour cell research. Nature Reviews. Cancer, 14, 623-631.

37. Melo, S. A., et al. (2015). Glypican-1 identifies cancer exosomes and detects early pancreatic cancer. Nature, 523, 177-182. 
38. Skog, J., et al. (2008). Glioblastoma microvesicles transport RNA and proteins that promote tumour growth and provide diagnostic biomarkers. Nature Cell Biology, 10, 1470-1476.

39. Stone, R. L., et al. (2012). Paraneoplastic thrombocytosis in ovarian cancer. The New England Journal of Medicine, 366, 610-618.

40. Mantur, M., Koper, O., Snarska, J., Sidorska, A., \& KruszewskaWnorowska, K. (2008). Evaluation of $\{\mathrm{PDGF}\}-\{\mathrm{AB}\}$ and $\{\mathrm{sP}\}-$ selectin concentrations in relation to platelet count in patients with colorectal cancer before and after surgical treatment. Polskie Archiwum Medycyny Wewnętrznej, 118, 345-350.

41. Cho, M. S., et al. (2012). Platelets increase the proliferation of ovarian cancer cells. Blood, 120, 4869-4872.

42. Bottsford-Miller, J., et al. (2015). Differential platelet levels affect response to taxane-based therapy in ovarian cancer. Clinical Cancer Research, 21, 602-610.

43. Ryningen, A., Apelseth, T., Hausken, T., \& Bruserud, Ø. (2006). Reticulated platelets are increased in chronic myeloproliferative disorders, pure erythrocytosis, reactive thrombocytosis and prior to hematopoietic reconstitution after intensive chemotherapy. Platelets, 17, 296-302.

44. Matowicka-Karna, J., Kamocki, Z., Polińska, B., Osada, J., \& Kemona, H. (2013). Platelets and inflammatory markers in patients with gastric cancer. Clinical \& Developmental Immunology, 2013, $1-6$.

45. Wang, R., et al. (2015). Electron cryotomography reveals ultrastructure alterations in platelets from patients with ovarian cancer. Proceedings of the National Academy of Sciences, 112, 1426614271.

46. Mantur, M., Kemona, H., Pietruczuk, M., \& Wasiluk, A. (2002). Does renal carcinoma affect the expression of P-selectin on platelets? Neoplasma, 49, 243-245.

47. Mantur, M., Kemona, H., Kozłowski, R., \& Kemona-Chetnik, I. (2003). Effect of tumor stage and nephrectomy on CD62P expression and sP-selectin concentration in renal cancer. Neoplasma, 50, 262-265.

48. Dymicka-Piekarska, V., Matowicka-Karna, J., Osada, J., Kemona, H., \& Butkiewicz, A. M. (2006). Changes in platelet CD 62P expression and soluble P-selectin concentration in surgically treated colorectal carcinoma. Advances in Medical Sciences, 51, 304-308.

49. Osada, J., Rusak, M., Kamocki, Z., Dabrowska, M. I., \& Kedra, B. (2010). Platelet activation in patients with advanced gastric cancer. Neoplasma, 57, 145-150.

50. Peterson, J. E., et al. (2012). VEGF, PF4 and PDGF are elevated in platelets of colorectal cancer patients. Angiogenesis, 15, 265-273.

51. Kamińska, J., et al. (2014). Does thrombopoiesis in multiple myeloma patients depend on the stage of the disease? Advances in Medical Sciences, 59, 166-171.

52. Meikle, C. K. S., et al. (2017). Cancer and thrombosis: the platelet perspective. Frontiers in Cell and Development Biology, 4, 147.

53. Cooke, N. M., et al. (2013). Increased platelet reactivity in patients with late-stage metastatic cancer. Cancer Medicine, 2, 564-570.

54. Angénieux, C., et al. (2016). Time-dependent decay of mRNA and ribosomal RNA during platelet aging and its correlation with translation activity. PloS One, 11, e0148064.

55. Balduini, C. L., et al. (1999). Relationship between size and thiazole orange fluorescence of platelets in patients undergoing high-dose chemotherapy. British Journal of Haematology, 106, 202-207.

56. Nilsson, R. J. A., et al. (2010). Rearranged EML4-ALK fusion transcripts sequester in circulating blood platelets and enable blood-based crizotinib response monitoring in non-small-cell lung cancer. Oncotarget, 1, 1066-1075.

57. Brown, G. T., \& McIntyre, T. M. (2011). Lipopolysaccharide signaling without a nucleus: kinase cascades stimulate platelet shedding of proinflammatory IL- $1 \beta$-rich microparticles. Journal of Immunology, 186, 5489-5496.
58. Chen, S., et al. (2016). Biological function and mechanism of MALAT-1 in renal cell carcinoma proliferation and apoptosis: role of the MALAT-1-Livin protein interaction. The Journal of Physiological Sciences. doi:10.1007/s12576-016-0486-8.

59. Huang, J., et al. (2016). MALAT1 promotes the proliferation and invasion of thyroid cancer cells via regulating the expression of IQGAP1. Biomedicine \& Pharmacotherapy, 83, 1-7.

60. Chen, D., et al. (2017). The role of MALAT-1 in the invasion and metastasis of gastric cancer. Scandinavian Journal of Gastroenterology, 52, 790-796.

61. Bian, D., Shi, W., Shao, Y., Li, P., \& Song, G. (2017). Long noncoding RNA GAS5 inhibits tumorigenesis via miR-137 in melanoma. American Journal of Translational Research, 9, 1509-1520.

62. Luo, G., et al. (2017). LncRNA GAS5 inhibits cellular proliferation by targeting P27Kip1. Molecular Cancer Research. doi:10.1158/ 1541-7786 MCR-16-0331.

63. Huang, T., et al. (2016). SNHG8 is identified as a key regulator of epstein-barr virus(EBV)-associated gastric cancer by an integrative analysis of lncRNA and mRNA expression. Oncotarget, 7, 8099081002 .

64. Zhao, L., et al. (2016). Long non-coding RNA SNHG5 suppresses gastric cancer progression by trapping MTA2 in the cytosol. Oncogene, 35, 5770-5780.

65. Zhao, L., et al. (2017). The lncRNA SNHG5/miR-32 axis regulates gastric cancer cell proliferation and migration by targeting KLF4. The FASEB Journal, 31, 893-903.

66. Russell, M. R., et al. (2015). CASC15-S is a tumor suppressor lncRNA at the 6p22 neuroblastoma susceptibility locus. Cancer Research, 75, 3155-3166.

67. Morenos, L., et al. (2014). Hypermethylation and down-regulation of DLEU2 in paediatric acute myeloid leukaemia independent of embedded tumour suppressor miR-15a/16-1. Molecular Cancer, $13,123$.

68. Kasar, S., et al. (2014). Therapeutic implications of activation of the host gene (Dleu2) promoter for miR-15a/16-1 in chronic lymphocytic leukemia. Oncogene, 33, 3307-3315.

69. Plé, H., et al. (2012). The repertoire and features of human platelet microRNAs. PloS One, 7, e50746.

70. Dangwal, S., \& Thum, T. (2013). MicroRNAs in platelet physiology and pathology. Hämostaseologie, 33, 17-20.

71. Dangwal, S., \& Thum, T. (2012). MicroRNAs in platelet biogenesis and function. Thrombosis and Haemostasis, 108, 599-604.

72. Edelstein, L. C., \& Bray, P. F. (2011). MicroRNAs in platelet production and activation. Blood, 117, 5289-5296.

73. Edelstein, L. C., et al. (2013). MicroRNAs in platelet production and activation. Journal of Thrombosis and Haemostasis, 11(Suppl 1), 340-350.

74. Gidlöf, O., et al. (2013). Platelets activated during myocardial infarction release functional miRNA, which can be taken up by endothelial cells and regulate ICAM1 expression. Blood, 121, 39083917, S1-26.

75. Gnatenko, D. V., et al. (2009). Class prediction models of thrombocytosis using genetic biomarkers. Blood, 115, 7-14.

76. Healy, A. M., et al. (2006). Platelet expression profiling and clinical validation of myeloid-related protein-14 as a novel determinant of cardiovascular events. Circulation, 113, 2278-2284.

77. Lood, C., et al. (2010). Platelet transcriptional profile and protein expression in patients with systemic lupus erythematosus: upregulation of the type I interferon system is strongly associated with vascular disease. Blood, 116, 1951-1957.

78. Raghavachari, N., et al. (2007). Amplified expression profiling of platelet transcriptome reveals changes in arginine metabolic pathways in patients with sickle cell disease. Circulation, 115, $1551-1562$

79. Rauschenberger, L., et al. (2016). Exosomal particles secreted by prostate cancer cells are potent mRNA and protein vehicles 
for the interference of tumor and tumor environment. Prostate, 76, 409-424.

80. Moscardó, A., Latorre, A., Santos, M. T., Bonanad, S., \& Vallés, J. (2015). Platelet function in malignant hematological disorders. Current Opinion in Oncology, 27, 522-531.

81. Pantel, K., \& Alix-Panabieres, C. (2016). Functional studies on viable circulating tumor cells. Clinical Chemistry, 62, 328-334.

82. Bray, P. F., et al. (2013). The complex transcriptional landscape of the anucleate human platelet. BMC Genomics, 14, 1.

83. Kissopoulou, A., Jonasson, J., Lindahl, T. L., \& Osman, A. (2013). Next generation sequencing analysis of human platelet
PolyA + mRNAs and rRNA-depleted total RNA. PloS One, 8, e81809.

84. Rowley, J. W., et al. (2011). Genome-wide RNA-seq analysis of human and mouse platelet transcriptomes. Blood, 118, e101-e111.

85. Simon, L. M., et al. (2014). Human platelet microRNA-mRNA networks associated with age and gender revealed by integrated plateletomics. Blood, 123, e37-e45.

86. Chen, L., et al. (2014). Transcriptional diversity during lineage commitment of human blood progenitors. Science, 345, 1251033.

87. Hrdlickova, B., et al. (2014). Expression profiles of long noncoding RNAs located in autoimmune disease-associated regions reveal immune cell-type specificity. Genome Medicine, 6, 88. 